\title{
EUROfusion
}

EUROFUSION WPS1-CP(16) 16512

J Fellinger et al.

\section{Integration of uncooled scraper elements and its diagnostics into Wendelstein 7-X}

Preprint of Paper to be submitted for publication in Proceedings of 29th Symposium on Fusion Technology (SOFT

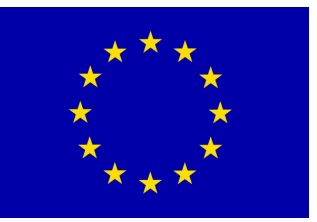

This work has been carried out within the framework of the EUROfusion Consortium and has received funding from the Euratom research and training programme 2014-2018 under grant agreement No 633053. The views and opinions expressed herein do not necessarily reflect those of the European Commission. 
This document is intended for publication in the open literature. It is made available on the clear understanding that it may not be further circulated and extracts or references may not be published prior to publication of the original when applicable, or without the consent of the Publications Officer, EUROfusion Programme Management Unit, Culham Science Centre, Abingdon, Oxon, OX14 3DB, UK or e-mail Publications.Officer@euro-fusion.org

Enquiries about Copyright and reproduction should be addressed to the Publications Officer, EUROfusion Programme Management Unit, Culham Science Centre, Abingdon, Oxon, OX14 3DB, UK or e-mail Publications.Officer@euro-fusion.org

The contents of this preprint and all other EUROfusion Preprints, Reports and Conference Papers are available to view online free at http://www.euro-fusionscipub.org. This site has full search facilities and e-mail alert options. In the JET specific papers the diagrams contained within the PDFs on this site are hyperlinked 


\title{
111Equation Chapter 1 Section 1Integration of uncooled scraper elements and its diagnostics into Wendelstein 7-X
}

\author{
Joris Fellinger $^{\mathrm{a}}$, Doug Loesser ${ }^{\mathrm{b}}$, Hutch Neilson ${ }^{\mathrm{b}}$, Arnie Lumsdaine ${ }^{\mathrm{c}}$, Dean McGinnis ${ }^{\mathrm{c}}$, Jeremy Lore, \\ Glen Wurden ${ }^{\mathrm{d}}$, Jörg Wendorf ${ }^{\mathrm{a}}$, Sören Klose ${ }^{\mathrm{a}}$, Uwe Wenzela ${ }^{\mathrm{a}}$, Klaus Grosser ${ }^{\mathrm{a}}$, Kerstin Rummel ${ }^{\mathrm{a}}$, \\ Hauke Hölbe ${ }^{\mathrm{a}}$, Thomas Sunn Pedersen ${ }^{\mathrm{a}}$, John Mitchell ${ }^{\mathrm{b}}$, Marc Sibilia ${ }^{\mathrm{b}}$, Han Zhang ${ }^{\mathrm{b}}$, Peter Titus ${ }^{\mathrm{b}}$ \\ and the W7-X team
}

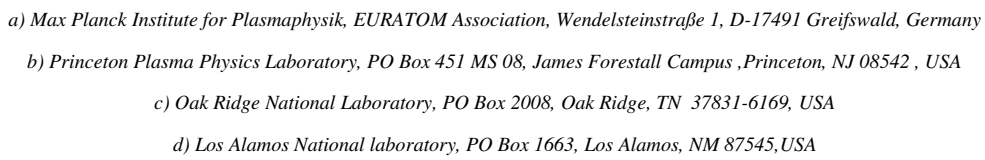

The modular stellarator Wendelstein 7-X in Greifswald (Germany) successfully started operation in 2015 with short pulse limiter plasmas. In 2017, the next operation phase (OP) OP1.2 will start once 10 uncooled test divertor units (TDU) with graphite armor will be installed. The TDUs allow for plasma pulses of $10 \mathrm{~s}$ with $8 \mathrm{MW}$ heating. OP2, allowing for steady state operation, is planned for 2020 after the TDUs will be replaced by 10 water cooled CFC armored divertors.

Due to the development of plasma currents like bootstrap currents in long pulse plasmas in OP2, the plasma could hit the edge of the divertor targets which has a reduced cooling capacity compared to the central part of the target tiles. To prevent overloading of these edges, a so-called scraper element can be positioned in front of the divertor, intersecting those strike lines that would otherwise hit the divertor edges. As a result, these edges are protected but as a drawback the pumping efficiency of neutrals is also reduced. As a test an uncooled scraper element with graphite tiles will be placed in two out of ten half modules in OP1.2. A decision to install ten water cooled scraper elements for OP2 is pending on the results of this test in OP1.2.

To monitor the impact of the scraper element on the plasma, Langmuir probes are integrated in the plasma facing surface, and a neutral gas manometer measures the neutral density directly behind the plasma facing surface. Moreover, IR and VIS cameras observe the plasma facing surface and thermocouples monitor the temperatures of the graphite tiles and underlying support structure.

This paper describes the integration of the scraper element and its diagnostics in Wendelstein 7-X.

Keywords: Wendelstein 7-X, strike lines, heat loads, scraper element, Langmuir probes, neutral gas manometer 


\section{Introduction}

\subsection{Background}

The modular stellarator Wendelstein $7-\mathrm{X}$ in Greifswald (Germany) successfully started operation in 2015 with short pulse limiter plasmas. In 2017, the next operation phase (OP) OP1.2 will start once 10 uncooled test divertor units (TDU) with graphite armor will be installed. The TDUs allow for plasma pulses of $10 \mathrm{~s}$ with $8 \mathrm{MW}$ heating. OP2, allowing for steady state operation, is planned for 2020 after the TDUs will have been replaced by 10 water cooled CFC armored divertors.

Due to the development of plasma currents like bootstrap currents in long pulse plasmas in OP2, strike line patterns onto the divertor migrate and the plasma could hit the edge of the divertor targets which has a reduced cooling capacity compared to the central part of the target tiles. To prevent overloading of these edges, a so-called scraper element can be positioned in front of the divertor, intersecting those strike lines that would otherwise hit the divertor edges. As a result, these edges are protected but as a drawback the pumping efficiency of neutrals is also reduced.

The scraper element is jointly developed by Oak Ridge National Laboratory, Princeton Plasma Physics Laboratory (both in the USA) and the Max-Planck institute of Plasma Physics, Germany (IPP) within the framework of the U.S.-EU bilateral agreement on collaboration in fusion research as agreed in the project agreement between IPP and the Department of Energy of the USA in which US researchers participate in the commissioning and scientific exploitation of W7-X.

\subsection{TDU Scraper Element (TDU-SE)}

As a test, uncooled scraper element with graphite tiles will be placed in two out of ten half modules in OP1.2. These are called TDU Scraper Elements (TDU-SEs). The mission of the TDU-SE is twofold:

- To assess whether the high heat-flux scraper element can effectively protect the ends of the divertor target in OP2.

- To determine how pumping effectiveness will be affected by the presence of a scraper element in OP2.

OP1.2 is split into OP1.2a without and OP1.2b with TDU-SEs. OP1.2a is planned to start in the first half of 2016 and in-vessel cables of TDU-SE diagnostics will already be installed behind the first wall. After a short break for the installation of the TDU-SEs, OP1.2b should start early 2017. The in-vessel layout of both phases is shown in fig. 1. A decision to install ten water cooled scraper elements for OP2 is pending on the results of OP1.2.

To monitor the impact of the scraper element on the plasma, Langmuir probes are integrated in the plasma facing surface, and a neutral gas manometer measures the neutral density directly behind the plasma facing surface. Moreover, IR and VIS cameras monitor the plasma facing surface and thermocouples record the temperatures of the graphite tiles and underlying support structure. The camera system is a joint development of Los Alamos national Laboratory in the USA and IPP.
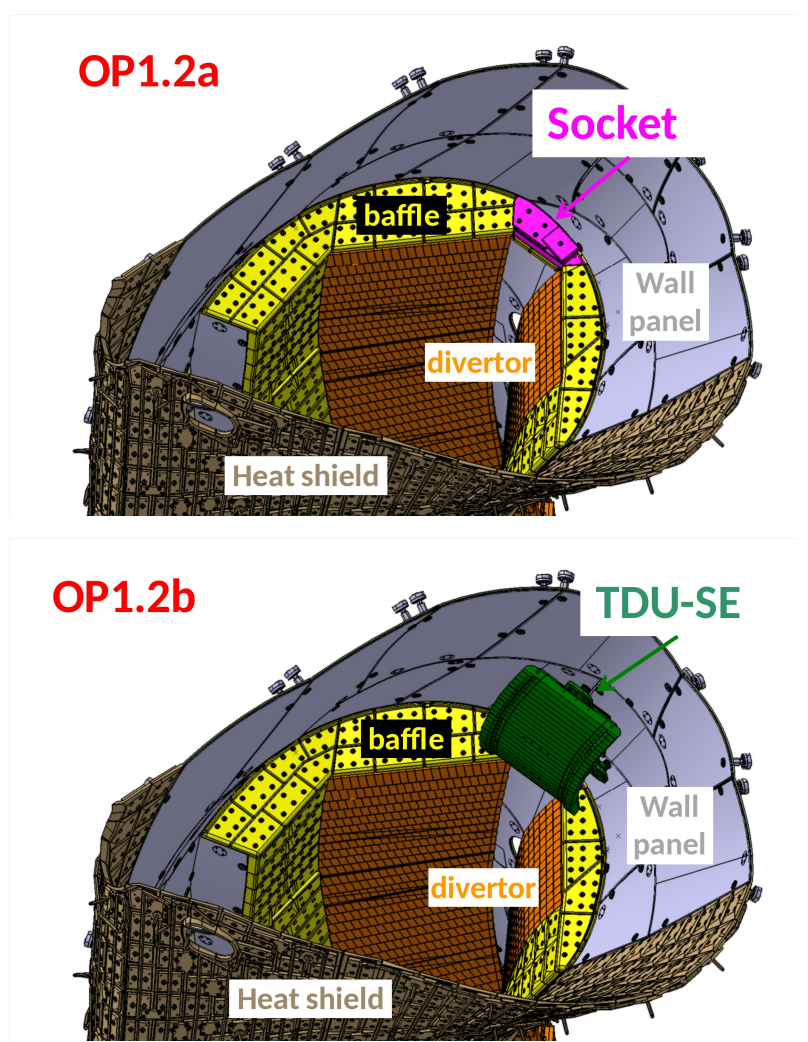

Fig. 1. In-vessel view without (OP1.2a) and with TDU-SE (OP1.2b).

\subsection{Outline}

This paper describes the ongoing activities to integrate the TDU-SE and its diagnostics into W7-X. In the next chapter, the design heat loads onto the TDU-SE are discussed which resulted from in-depth analyses of electromagnetic configurations for OP1.2 to mimic slowly developing strike line patterns during long time pulses in OP2. In chapter 3, the support system of the TDU-SE is discussed on top of the existing stainless steel wall panels. The diagnostics of the TDU-SE and the installation of the in-vessel routing of their cables up to the vacuum feed-through are presented in chapter 4 .

\section{Heat loads}

\subsection{Divertor edge overload}

The water cooled divertor that is planned for OP2 has a reduced design load at the edge near the pumping gap due to the local U-shape of the cooling circuit, see green area in fig. 2.

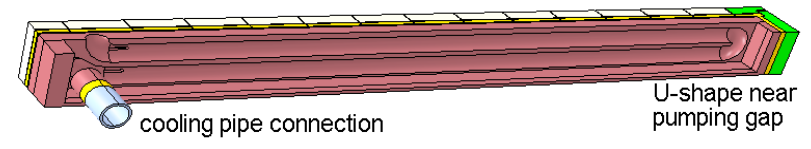

Fig. 2. Cross sectional view of target element of the water cooled divertor of OP2. The bottom part of the cooling block is removed to show the shape of the cooling loop 
In long pulse operation in OP2, plasma currents like bootstrap currents are expected to develop over 1-2 minutes, which change the electromagnetic configuration. It mainly leads to a poloidal shift of the strike line patterns onto the divertor. Consequently, the edges might get overloaded, especially in the transient phase of bootstrap current growth in high performance scenarios. A scraper element intercepts the strike lines that would otherwise hit the edges, see fig 3 .

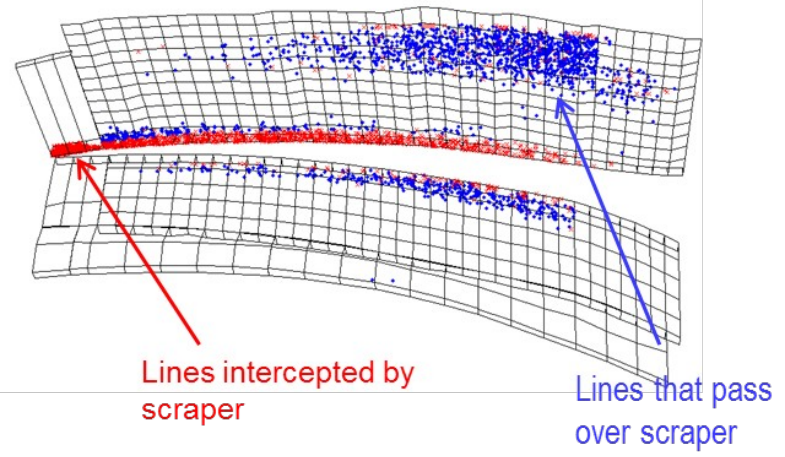

Fig. 3. Strike patterns on the horizontal and vertical divertor and associated baffles at a bootstrap current of $22 \mathrm{kA}$. Blue points are field lines that pass over the scraper element, red points indicate field lines that would hit the target if the scraper element was removed [Lore: 2014]

However, bootstrap currents and corresponding poloidal shifts of strike line patterns cannot fully develop within the short pulses $(\sim 10 \mathrm{~s})$ of OP1.2. As a result, less than $0.05 \%$ of the heating power is deposited onto the TDU-SE in all reference configurations of $\mathrm{W} 7-\mathrm{X}$ in OP1.2 Hölbe 2016]. So, the TDU-SE is only loaded in electromagnetic configurations that are dedicated to test the TDU-SE.

\subsection{Mimic scenarios}

To measure the impact of the TDU-SE, mimic scenarios were developed for OP1.2 in which currents in the main superconducting (non) planar coils (NPC \& PC) and invessel control coils (CC) are adapted to mimic the expected strike line patterns on the divertor and scraper elements during bootstrap current growth phase of a pursued high performance scenario of OP2 [Hölbe: 2016], see table 1.

Table 1. Relative coil currents in reference scenario of OP2 and mimic scenarios of OP1.2 to mimic bootstrap currents of 0 , 22 and $43 \mathrm{kA}$.

\begin{tabular}{|c|c|c|c|c|c|c|c|c|c|}
\hline $\mathrm{OP}$ & NPC1 & NPC2 & NPC3 & NPC4 & NPC5 & PCA & PCB & $\mathrm{CC} 1$ & $\mathrm{CC} 2$ \\
\hline 2 & 1 & 0.989 & 1.01 & 1.114 & 1.124 & 0.247 & -0.08 & 0 & 0 \\
\hline $1.2-0$ & & & & & & 0.22 & -0.08 & & \\
\hline $1.2-22$ & 0.96 & 0.95 & 0.97 & 1.07 & 1.08 & 0.17 & -0.13 & -0.015 & 0.015 \\
\hline$\overline{1.2-43}$ & & & & & & 0.12 & -0.18 & & \\
\hline
\end{tabular}

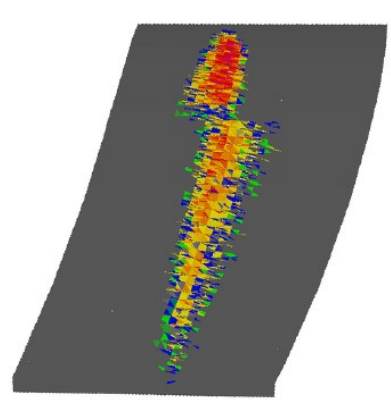

(a) SE, mimic $22 \mathrm{kA}$

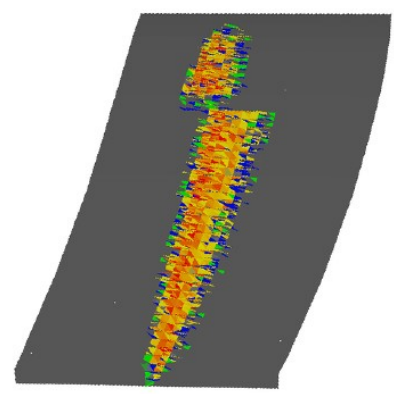

(c) SE, real $22 \mathrm{kA}$

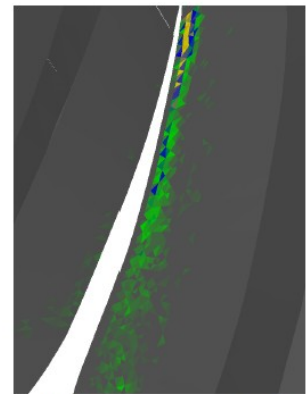

(b) Divertor, mimic $22 \mathrm{kA}$

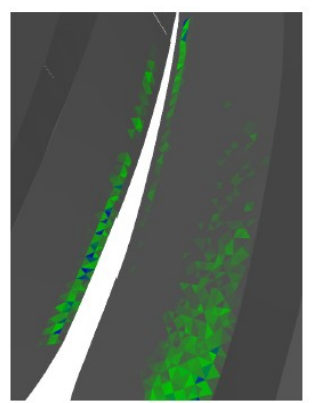

(d) Divertor, real $22 \mathrm{kA}$

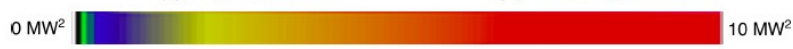

Fig. 4. Comparison of strike line patterns between mimic scenario in OP1.2 (top) and reference scenario in OP2 (bottom)

\subsection{Design heat load on TDU-SE}

A principle step in the design of the TDU-SE is the definition of the heat loads onto the TDU-SE. The design heat load is exclusively defined by the heating power in the mimic scenario since the TDU-SE is barely hit by strike lines in any of the reference scenarios, see table $\mathrm{X}$.

The heating power in the mimic configuration was reduced from the OP1.2 maximum of $80 \mathrm{MJ}$ to $2 \mathrm{MW}$ for $6.25 \mathrm{~s}(12.5 \mathrm{MJ})$. It allows for a simplification of the TDU-SE design like change of the support bars in the graphite tiles from TZM to steel [3 Loesser: 2016]. The design load was simplified to a roof shaped distribution, see fig. 5 with a peak of $8 \mathrm{MW} / \mathrm{m}^{2}$.
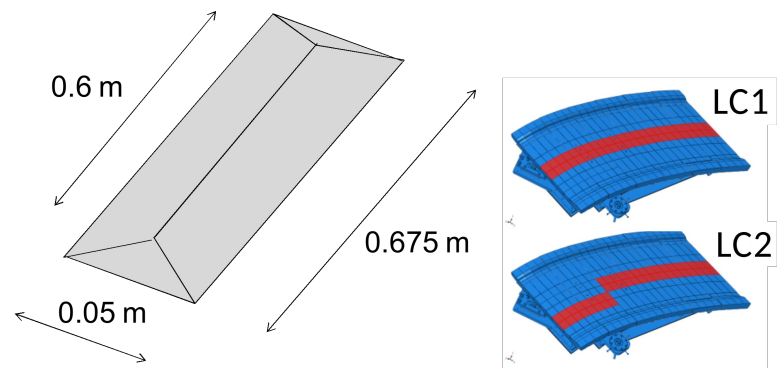

Fig. 5. Design heat load onto TDU-SE.

If was proven that the heating of the plasma facing surface under this reduced load can clearly be identified by the IR cameras, see fig. 6 .

Similarly, it was demonstrated that Langmuir probes will be loaded within their operational regime assuming an electron density of $10^{19}-10^{20} \mathrm{~m}^{-3}$. The electrical power of the Langmuir probes is assumed to be proportional to the heat load and exposed surface area of the Langmuir 
probes. For the $\varnothing_{5} \mathrm{~mm}$ probes, the expected ion saturation current vs. floating potential is given in fig. 7 .
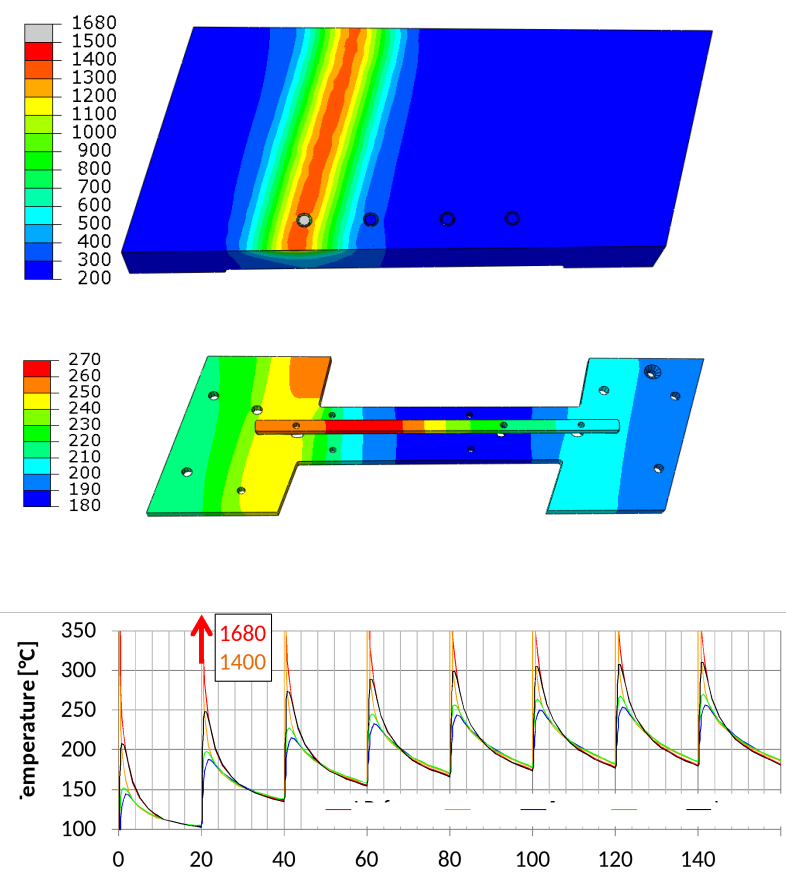

Fig. 6. Distribution of maximum temperature $\left[{ }^{\circ} \mathrm{C}\right]$ in graphite tile (top) and steel components (middle) and history at highest loaded spots in Langmuir probes, tiles and steel parts (bottom) over 8 pulses of $2 \mathrm{MW}$ for $6.25 \mathrm{~s}$ with 20 minutes dwell time.

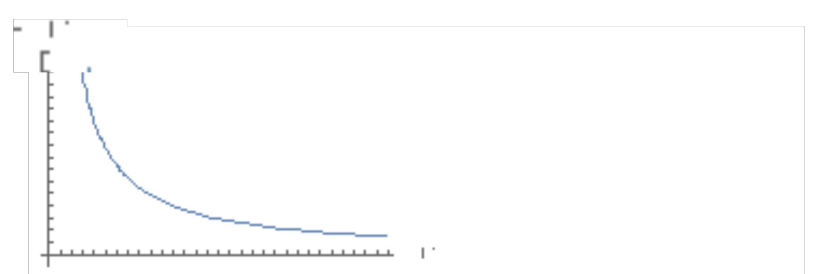

Approximate measurement range

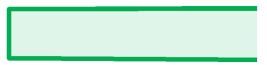

Fig. 7. Ion saturation current versus floating potential for Langmuir probes of $5 \mathrm{~mm}$ diameter loaded with $8 \mathrm{MW} / \mathrm{m}^{2}$.

Finally it was also verified whether the neutral gas manometer could measure the impact of the TDU-E on neutrals production at the reduced heating scenario. Assuming a neutral density behind the TDU-SE of $1 / 50-1 / 5$ of the electron density at the edge and an average temperature of the neutrals matching that of the backside of the TDU-SE of 300-600 K, the neutral pressure $p_{0}=n_{0} k_{B} T$ is expected to be in the range of $300 / 50 \times 10^{19} k_{B}<p_{0}<600 / 5 * 0^{20} k_{B}$

$10^{-5}-10^{-3}$ mbar, which is well within the measurement range of $10^{-6}-10^{-1}$ mbar.

\section{Installation considerations}

\subsection{Support system}

The decision to install two TDU-SEs was made several years after the design of the plasma facing components was finished. At the location of the TDU-SE, stainless steel wall panels are protecting the plasma vessel wall from the plasma radiation. The TDU-SE will be installed onto these panels using a ball mount extension onto the wall panel supports, see fig. 8. To prevent thermal stress induced by restrained thermal deformations, the TDU-SE can rotate on the ball mount of each support. Moreover, the base plate of the TDU-SE is has an in-plane fixation at ball mount 1, can freely expand at ball mount 2 in the direction of ball mount 1 but is fixed in the perpendicular in-plane direction and has no in-plane restraints at ball mount 3 .

As fixed as well as flexible wall panel supports are used to mount the wall panels throughout W7-X (to prevent thermal stress from restrained thermal expansion of the wall panels), it was ensured that support 1 and 2 were of the fixed type to obtain a well defined position of the TDU-SE.

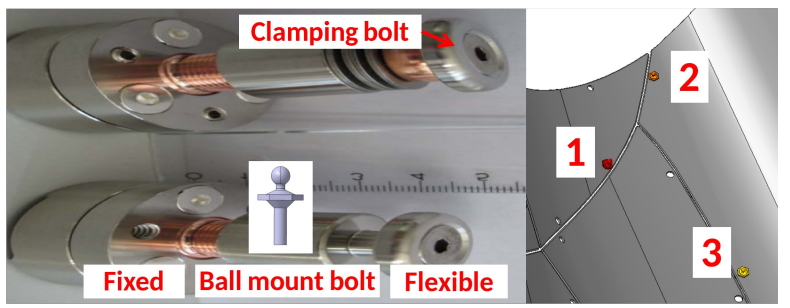

Fig. 8. Fixed and flexible wall panels support and screw that is exchanged with ball mount to bear the TDU-SE (left) and position of 3 TDU-SE supports (right).

The TDU-SE will be installed in two parts, i.e. a base plate of stainless steel of $12 \mathrm{~kg}$ that allows for the alignment and a first wall of $27 \mathrm{~kg}$ of the graphite tiles on a stainless steel back plate which in module 5 also include the Langmuir probes and neutral gas manometer. The TDU-SE must be installed within $\pm 1.5 \mathrm{~mm}$ tolerance in all directions relative to the TDU divertor and an angular tolerance of $0.2^{\circ}$ and $0.5^{\circ}$ about the poloidal and toroidal axis respectively. The supports of the base plate can be adjusted $\pm 7 \mathrm{~mm}$ in all directions to accommodate deviations of the ball mount positions relative to the TDU divertor. The first wall is connected with a rigid ball mount in the middle and two flexible supports near the end allowing for thermal expansion. Once the base plate is aligned, the first wall position is defined as well.

The installation of the TDU-SE must be constrained to very limited time in the short break between OP1.2a and OP1.2b. To anticipate an installation without venting of the cryostat (i.e. with 1 bar pressure difference from the inside to the outside of the plasma vessel compared to no pressure difference during operation) the deformation of the wall panel supports 1-3 relative to the TDU divertor were measured after OP1.1 before and after venting of the cryostat. The pressure onto the vessel caused relative displacements of 0.4-1.1 mm. These displacements are taken into account when the target position of the 
TDU-SE will be defined. Additional local deformations of the plasma vessel due to vacuum loads on port flanges are neglected which is supported by FE analysis of the cryostat system [6: van Eeten].

\section{Diagnostic integration}

Langmuir probes and a neutral gas manometer are intergrated in the TDU-SE in module 5. To facilitate the connection of the Langmuir probes and neutral gas manometer to the electronics outside the plasma vessel, a socket was installed before OP1.2a onto the toroidal closure in the vicinity of the TDU-SE. Kapton insulated copper cables are routed from the socket to the vacuum flange behind plasma facing components and protected in a copper tube against electron cyclotron stray radiation. During OP1.2a, the socket and cable tray at the toroidal closure are protected by a graphite tile, see fig. 9 for details and fig 1. During OP1.2b, the socket and cable tray are shielded by the TDU-SE.

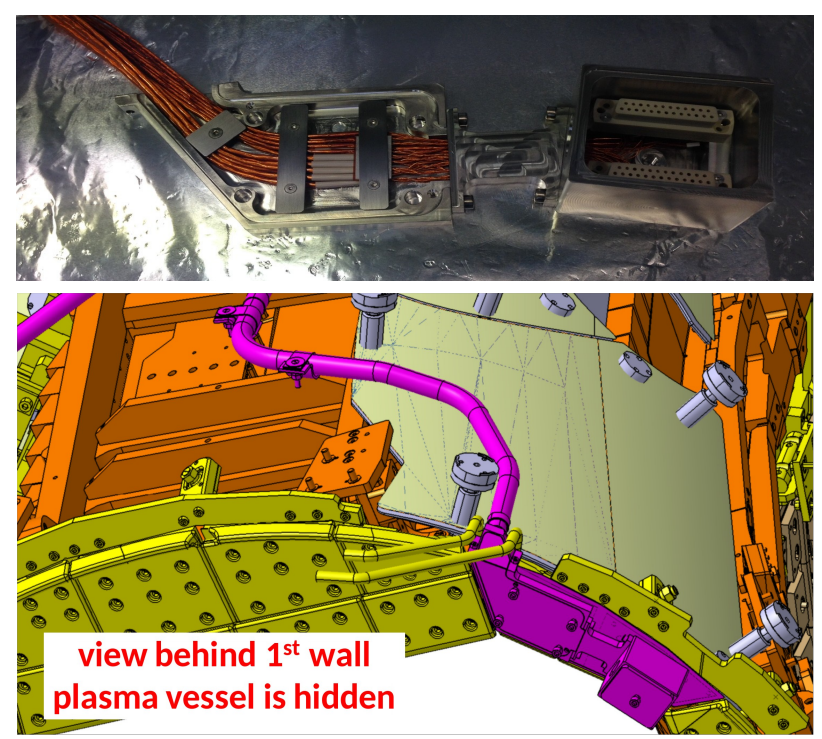

Fig. 9. Cable box with Kapton cables and socket (top) and detailed view of the routing of the cables behind the plasma facing components (bottom).

Metal coated cables with heat resistant ceramic insulation are used on the first wall of the TDU-SE between the diagnostics to the plug that is temporarily mounted onto the back plate. During installation, once the first wall is fixed onto the base plate at the central ball mount, the plug can be inserted into the socket before two flexible supports are being fixed. The installation sequence will be tested and optimized in the test module of the plasma vessel during OP1.2a.

\section{Acknowledgments}

This work has been carried out within the framework of the EUROfusion Consortium and has received funding from the Euratom research and training programme 2014-2018 under grant agreement No 633053. The views and opinions expressed herein do not necessarily reflect those of the European Commission.

The funding from the US Department of Energy for the contribution of Oak Rigde national Laboratory, Princeton Plasma Physics laboratory and Los Alamos National Laboratory to the TDU-SE project is gratefully acknowledged.

\section{References}

[1] H. Hölbe: Control of the magnetic topology and plasma exhaust in the edge region of Wendelstein 7-X: A numerical study, IPP Report 18/4, February 2016

[2] H. Hölbe et al.: Access to edge scenarios for testing a SE in early operation phases of W7-X, Nuclear Fusion 56 (2), February 2016

[3] D.Loesser et al.: Detailed design and analysis of W7-X scraper element, this conference, poster P2.121

[4] J. Boscary et al.: Development activities of the high heat flux scraper element, Fusion Engineering and Design 98-99, October 2015, pp. 1231-1234

[5] J. Lore et al: Design and Analysis of Divertor SEs for the W7-X Stellarator, IEEE Transactions on Plasma Science 42 (3), March 2014

[6] P. van Eeten et al.: Features and analyses of W7-X cryostat system FE model, Fusion Engineering and Design 96-97, October 2015, pp. 369-372 